\title{
Prueba hipergeométrica aleatorizada
}

\author{
Randomized Hypergeometric Test \\ Jorge Eduardo Ortiz Pinilla a \\ jorgeortiz@usantotomas.edu.co
}

\begin{abstract}
Resumen
Las pruebas aleatorizadas se encuentan bien documentadas teóricamente, pero son poco usadas en las aplicaciones prácticas. Las computadoras han abierto las puertas a los métodos basados en la generación de números aleatorios para construir procedimientos de análisis estadísticos. En este artículo se presenta en detalle la prueba hipergeométrica aleatorizada para ilustrar su utilidad en cuanto al acercamiento del tamaño de la pruebas con estadísticas discretas a los niveles de significación usuales.
\end{abstract}

Palabras clave: pruebas aleatorizadas, prueba hipergeométrica, tamaño de la prueba, nivel de significación, error tipo I.

\begin{abstract}
Randomized tests are well documented in theory, but are rarely used in practical applications. With the computers, many methods based on random number generation to build statistical analysis procedures have been developed. In this article we present in detail the hypergeometric randomized test to illustrate its usefulness in terms of approaching the size of hypotheses tests based on discrete statistics to the usual significance levels.
\end{abstract}

Key words: Randomized Tests, Hypergeometric Test, Test Size, Significance Level, Type I Error.

\section{Introducción}

A pesar de las controversias generadas por el uso del valor-p para las pruebas de hipótesis en el contexto de Neyman-Pearson, el software actual lo impone convirtiéndolo en el elemento principal para definir la regla de decisión, mediante su

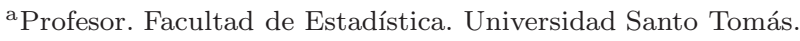


comparación con el nivel de significación $\alpha$. Este uso se justifica por su equivalencia con la regla de decisión basada en puntos críticos. Bickel \& Docksum (1977, p. 173) proponen verlo como una versión estandarizada de la estadística original de prueba para definir la regla de decisión de una manera única:

Rechazar $H_{0}$ si el valor-p es inferior o igual al nivel de significación, $\alpha$, fijado previamente por el investigador. En caso contrario, la hipótesis nula no se rechaza.

Con el nivel de significación se establece un control sobre la probabilidad de cometer el error tipo I (rechazar equivocadamente $H_{0}$ ). Sin embargo, estos dos valores no siempre coinciden. Los autores mencionados arriba llaman el tamaño de la prue$b a$ a la mayor probabilidad de rechazar $H_{0}$ siendo cierta que se puede tener con la prueba definida.

Cuando la estadística de prueba es discreta no siempre es posible encontrar un cuantil de la distribución que corresponda exactamente al nivel de significación que el investigador establece y, además, frecuentemente el nivel de significación y el tamaño de la prueba son diferentes. El siguiente ejemplo con la distribución binomial ilustra esta situación.

Supongamos que para probar $H_{0}: p \leq 0.5$ versus $H_{1}: p>0.5$ con un nivel de significación $\alpha=0.05$ se toma una muestra de tamaño $n=17$ y supongamos además que el verdadero valor de $p$, aunque desconocido para el investigador, es 0.45 . Como $p=0.45<p_{0}=0.5$, entonces $H_{0}$ es verdadera. El siguiente código en $\mathrm{R}$ muestra las distribuciones de probabilidad de $X \sim \operatorname{Bin}(n, p)$, en la condición real con $p=0.45$ y en la condición de $p=0.5$ que utiliza el investigador para establecer su regla de decisión (rechazar $H_{0}$ si $x_{o b s} \geq 13$ o si el valor-p $\left.=P\left(X \geq x_{o b s} ; p=0.5\right) \leq \alpha\right)$ :

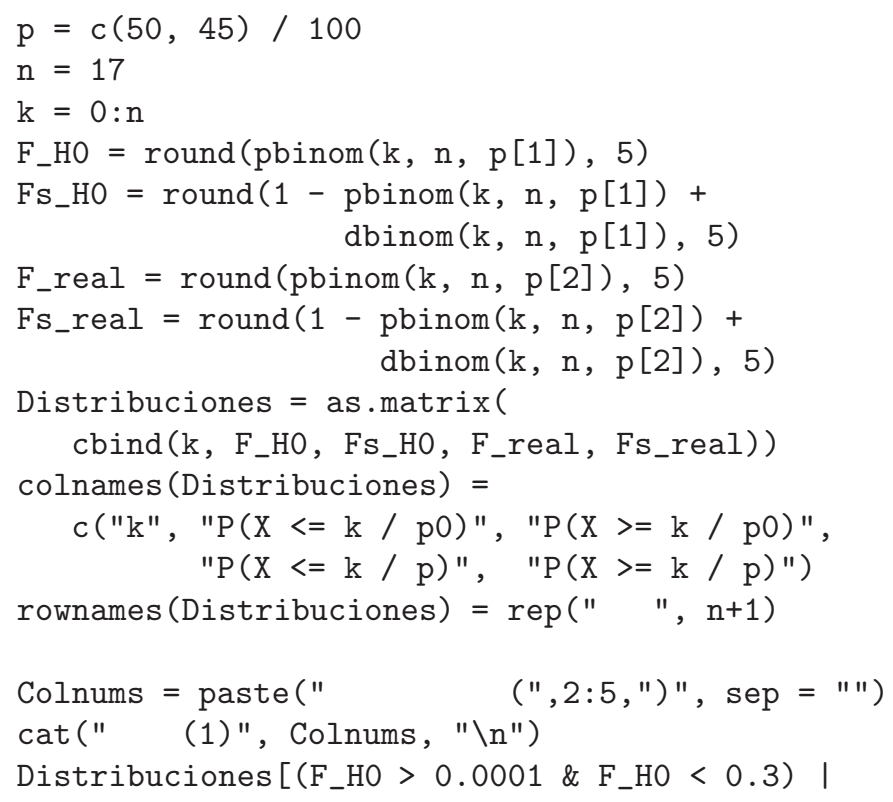


(Fs_real $>0.0001 \&$ Fs_real $<0.3)$, ]

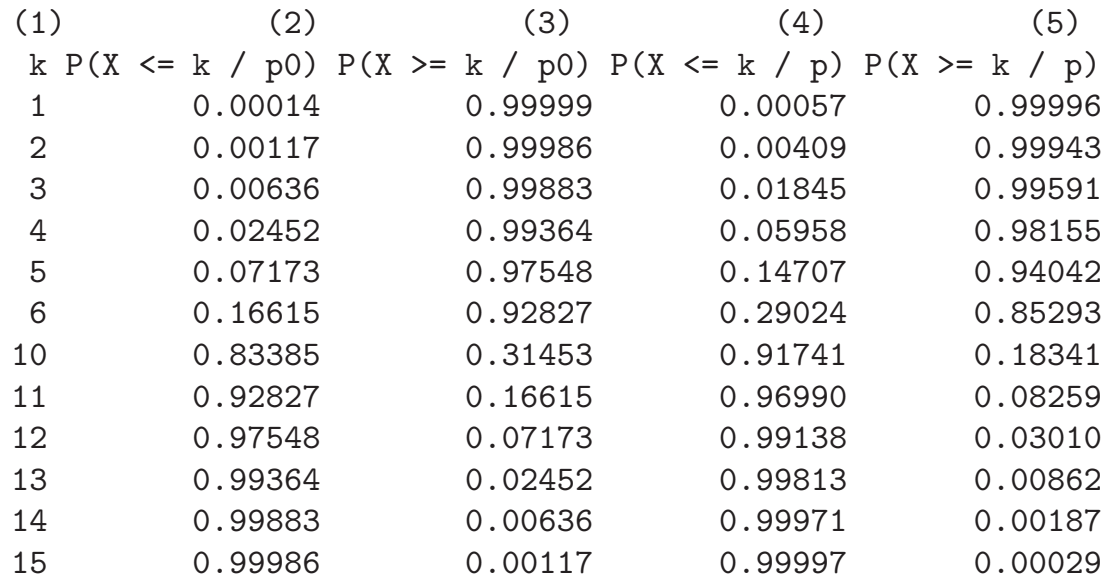

En este ejemplo, el nivel de significación es de 0.05. En las columnas (3) y (5) de la tabla anterior puede comprobarse que el tamaño de la prueba es de 0.0245 y que la verdadera probabilidad de rechazar $H_{0}$ (verdadera) es igual a 0.009. En la práctica esta última probabilidad se desconoce como consecuencia del desconocimiento del verdadero valor de $p$, pero mientras $H_{0}$ sea cierta se encontrará acotada, tanto por el nivel de significación, como por el tamaño de la prueba.

$\mathrm{Si}$ el investigador hubiera querido mostrarse un poco más tolerante con el error tipo I, habría podido escoger como nivel de significación un valor de 0.06 o 0.07 y, en otras circunstancias, se hubiera mostrado menos tolerante tomando 0.04 o 0.03 . Sin embargo, para todos estos casos, el tamaño de la prueba es el mismo 0.0245 y la regla de decisión, aparentemente diferente, en realidad no ha cambiado. En efecto, se trata de la mínima cota superior de la probabilidad real de cometer el error tipo I. De ahí su importancia.

Ante el desconocimiento de $p$ y de la probabilidad de cometer el error tipo I, lo ideal es que se establezcan las condiciones para que el nivel de significación y el tamaño de la prueba coincidan y así, el investigador tenga una mejor idea del control que ejerce sobre esta probabilidad.

\section{La prueba hipergeométrica}

De manera clásica, la distribución hipergeométrica se encuentra cuando se seleccionan sin reposición $n$ de los $N$ elementos que contiene una urna, considerando que $N_{A}$ del total tienen una determinada propiedad $A$. Si $X$ es la variable aleatoria que cuenta los elementos con $A$ en la muestra, entonces, la probabilidad de encontrar $X=x$ es igual a: 


$$
\operatorname{Pr}(X=x)=\frac{\left(\begin{array}{c}
N_{A} \\
x
\end{array}\right)\left(\begin{array}{c}
N-N_{A} \\
n-x
\end{array}\right)}{\left(\begin{array}{l}
N \\
n
\end{array}\right)}
$$

y se define la función de distribución de $X$ como

$$
F_{X}\left(x_{o}\right)=\sum_{x \leq x_{o}} \operatorname{Pr}(X=x)
$$

Los parámetros de carácter poblacional de esta distribución son $N$ y $N_{A}$ y los problemas inferenciales usuales son acerca de sus valores. Nos centraremos en las pruebas de hipótesis unilaterales relacionadas con $N_{A}$ suponiendo $N$ conocido. Entonces, si una población $\Omega$ de tamaño conocido $N$ se encuentra dicotomizada por una propiedad $A$, pero se deconoce la cantidad $N_{A}$ de elementos que la tienen, se pueden establecer y probar hipótesis sobre su valor, por ejemplo:

$$
\begin{aligned}
& H_{0}: N_{A} \leq N_{0} \quad \text { (donde } N_{0} \text { es un valor conocido) } \\
& H_{1}: N_{A}>N_{0}
\end{aligned}
$$

Una vez fijados el tamaño de muestra $n$ y un conjunto de valores $x_{1}, x_{2}, \ldots, x_{n}$, donde $x_{i}=0$ o $x_{i}=1$, y denotando como $X_{i}$, la variable aleatoria asociada a la $i$-ésima observación de ensayos de Bernoulli sin reposición, la variable $X=$ $\sum_{i=1}^{n} X_{i}$ tiene distribución hipergeométrica con parámetros $\left(N, N_{A}, n\right)$, dada por (11). Además, independientemente de las posiciones de los 0 y de los 1 en la secuencia observada, si $x=\sum_{i=1}^{n} x_{i}$, la función de verosimilitud para $N_{A}$ es:

$$
\begin{aligned}
L\left(N_{A}\right) & =\operatorname{Pr}\left(X_{1}=x_{1}, X_{2}=x_{2}, \ldots, X_{n}=x_{n} \mid N_{A}\right) \\
& =\frac{\frac{N_{A} !}{\left(N_{A}-x\right) !} \frac{\left(N-N_{A}\right) !}{\left(\left(N-N_{A}\right)-(n-x)\right) !}}{\frac{N !}{(N-n) !}} \\
& =\frac{1}{\left(\begin{array}{l}
n \\
x
\end{array}\right)} \operatorname{Pr}\left(X=x \mid N_{A}\right)
\end{aligned}
$$

Esta función es monótona y Rohatgi (1977) demuestra que la prueba basada en rechazar $H_{0}$ en favor de $H_{1}$ si $x \geq k$ es uniformemente más potente, con $k$ definido en función del nivel de significación. 
Supongamos, por ejemplo, que de una población $\Omega$ de tamaño $N=5000$ se seleccionan sin reemplazo $n=51$ elementos para probar con un nivel de significación de 0.05 las siguientes hipótesis:

$$
\begin{aligned}
& H_{0}: N_{A} \leq 1000 \\
& H_{1}: N_{A}>1000
\end{aligned}
$$

Con el código en $\mathrm{R}$ que se presenta a continuación se obtiene la tabla de la distribución $F_{X}$. Se agregaron las columnas con la función de distribución superior, $F s_{X}(x)=\operatorname{Pr}(X \geq x)$ y las correspondientes a $N_{A}=1500$, para ilustrar el cálculo de la función de potencia para este valor.

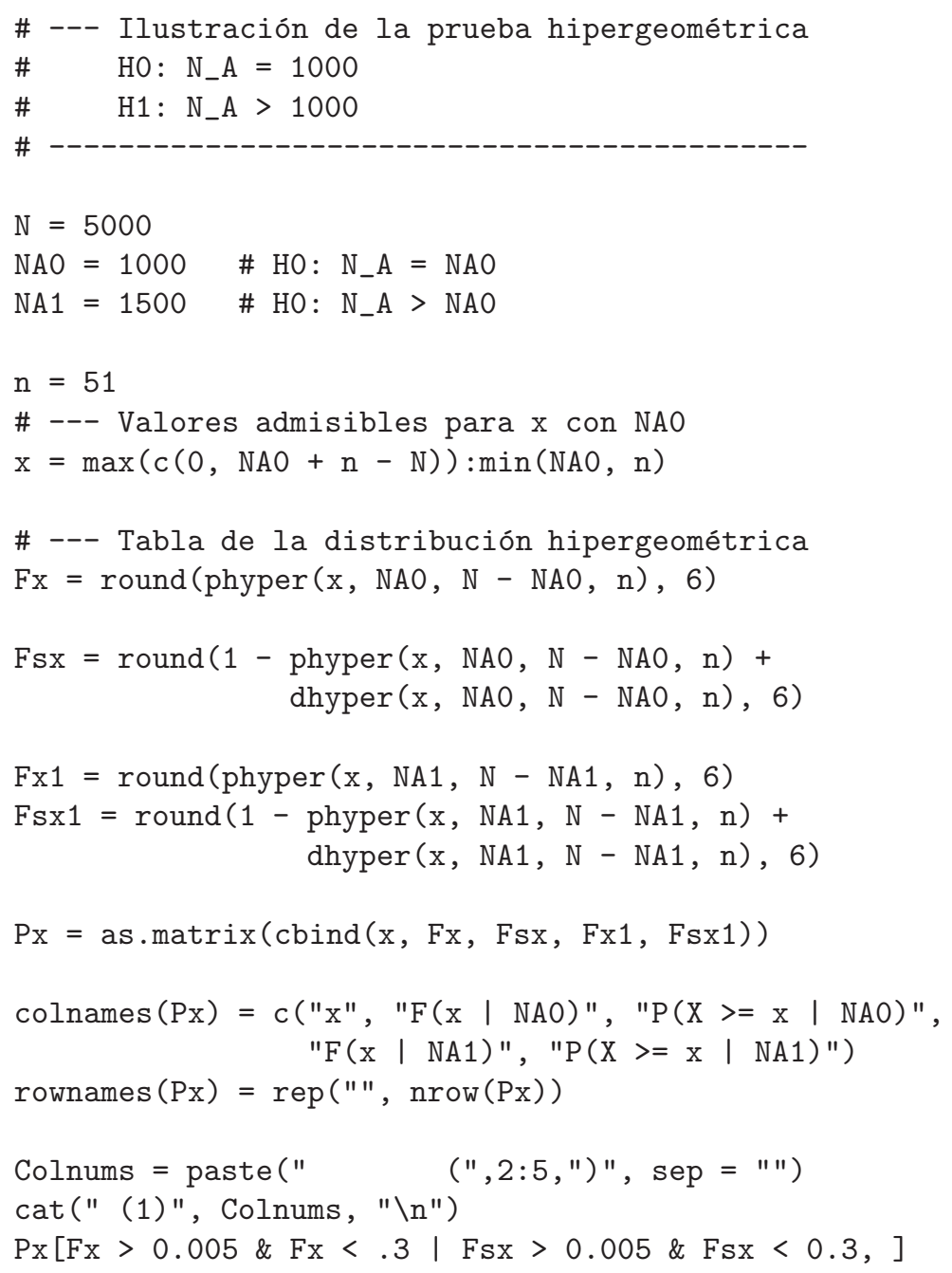




\begin{tabular}{|c|c|c|c|c|}
\hline (1) & (2) & (3) & (4) & (5) \\
\hline $\mathrm{x}$ & $F(x \mid N A 0) P(X>$ & $\mathrm{x} \mid \mathrm{NAO})$ & $\mathrm{F}(\mathrm{x} \mid \mathrm{NA1}) \quad \mathrm{P}(\mathrm{X}>=$ & $\mathrm{x} \mid \mathrm{NA1})$ \\
\hline 4 & 0.015537 & 0.995373 & 0.000122 & 0.999978 \\
\hline 5 & 0.041368 & 0.984463 & 0.000530 & 0.999878 \\
\hline 6 & 0.091192 & 0.958632 & 0.001882 & 0.999470 \\
\hline 7 & 0.171670 & 0.908808 & 0.005640 & 0.998118 \\
\hline 8 & 0.282748 & 0.828330 & 0.014566 & 0.994360 \\
\hline & 0.875480 & 0.205651 & 0.295300 & 0.803572 \\
\hline 4 & 0.930325 & 0.124520 & 0.410536 & 0.704700 \\
\hline 5 & 0.963976 & 0.069675 & 0.532475 & 0.589464 \\
\hline 16 & 0.982785 & 0.036024 & 0.650058 & 0.467525 \\
\hline 17 & 0.992393 & 0.017215 & 0.753709 & 0.349942 \\
\hline 18 & 0.996890 & 0.007607 & 0.837455 & 0.246291 \\
\hline
\end{tabular}

Los valores de la tabla no permiten construir una prueba con un nivel de significación usual. Por ejemplo, para $\alpha=0.05$, el punto crítico es 16 y el tamaño de la prueba es entonces 0.036024 .

Se tienen dos opciones generales:

1. Modificar el nivel de significación, acogiéndose a las limitaciones de la distribución y tomando alguno de los que se encuentran en la función de distribución. En el ejemplo, tomar como nivel de significación $\alpha=0.036024 \mathrm{u}$ otro que se considere conveniente.

2. Aplicar procedimientos adicionales para modificar la regla de decisión buscando igualar el tamaño de la prueba al nivel de significación o, al menos, aproximarlo.

La primera opción es la más directa, pero, por una parte, el software disponible no la ofrece con la misma facilidad con que arroja los resultados acompañados del valor-p como elemento determinante de la regla de decisión; y, por otra, los mismos investigadores pueden sentir que, de no utilizar una regla estándar, propician condiciones de poca comparabilidad de unos resultados a otros si el nivel de significación cambia en cada caso.

La segunda opción se encuentra bastante documentada desde el punto de vista teórico con lo que se ha definido como las pruebas aleatorizadas (randomized tests), pero su implementación práctica sólo está en manos de investigadores estadísticos y ha sido de poco recibimiento en las aplicaciones prácticas. Tal vez el argumento más utilizado en su contra es el mencionado por Mood et al. (1974) como el hecho de "dejar la decisión en manos de una variable completamente ajena a los datos obtenidos con las muestras observadas".

Una tercera opción, la más común, consiste en dejar que el investigador dé un valor para el nivel de significación, que por lo general es $\alpha=0.05$ y tome la decisión de 
rechazar $H_{0}$ cuando el valor-p resulte menor o igual que $\alpha$, sin tomar conciencia de los inconvenientes mencionados antes.

El propósito de este trabajo es ilustrar el método de pruebas aleatorizadas aplicado a la prueba hipergeométrica sobre el parámetro $N_{A}$.

\subsection{Prueba hipergeométrica aleatorizada}

Sea $k_{1-\alpha}$ el cuantil de orden $1-\alpha$ de la distribución $F_{X}$, es decir:

$$
k_{1-\alpha}=\min \left\{k \mid 0 \leq k \leq n, F_{X}(k) \geq 1-\alpha\right\}
$$

El mínimo valor que satisface la condición $F_{X}(k) \geq 1-\alpha$ es $k_{1-\alpha}$. Además, como $k_{1-\alpha}-1<k_{1-\alpha}$, entonces $\operatorname{Pr}\left(X \leq k_{1-\alpha}-1\right)<1-\alpha$ y por lo tanto,

$$
\operatorname{Pr}\left(X \geq k_{1-\alpha}\right)>\alpha
$$

Esto implica que con distribuciones discretas el cuantil $k_{1-\alpha}$ no pertenece a la región de rechazo de $H_{0}$ en pruebas unilaterales derechas. Por otra parte, como $F_{X}\left(k_{1-\alpha}\right) \geq 1-\alpha$, entonces

$$
\operatorname{Pr}\left(X>k_{1-\alpha}\right) \leq \alpha
$$

De las relaciones (4) y (5), el punto $k_{1-\alpha}$ es donde se supera el valor de $\alpha$ en la cola derecha. Entonces una opción para que el tamaño de la prueba sea exactamente $\alpha$ consiste en condicionar la inclusión de $k_{1-\alpha}$ en la región de rechazo de $H_{0}$ con un factor aleatorio que permita lograrlo.

Por (5), los valores $x>k_{1-\alpha}$ hacen rechazar $H_{0}$ sin condiciones adicionales. La inclusión de $k_{1-\alpha}$ se hará mediante el uso de una variable $U$ con distribución uniforme, independiente de $X$, cuya pertenencia a la región de rechazo de $H_{0}$ permita igualar el tamaño de la prueba con el nivel de significación $\alpha$. Así, $H_{0}$ será rechazada si

$$
\left(x>k_{1-\alpha}\right) \text { o si }\left(x=k_{1-\alpha} \text { y } u \in A_{R}\right)
$$

donde $x$ y $u$ son observaciones de $X$ y $U$, respectivamente, y $A_{R} \subset[0,1)$ es un intervalo que se determinará enseguida.

$$
\begin{aligned}
\alpha & =\operatorname{Pr}\left(\text { Rechazar } H_{0} \mid H_{0}\right) \\
& =\operatorname{Pr}\left(X>k_{1-\alpha}\right)+\operatorname{Pr}\left(X=k_{1-\alpha}, U \in A_{R}\right) \\
& =\left(1-F_{X}\left(k_{1-\alpha}\right)\right)+\operatorname{Pr}\left(X=k_{1-\alpha}\right) \operatorname{Pr}\left(U \in A_{R}\right)
\end{aligned}
$$


luego

$$
\operatorname{Pr}\left(U \in A_{R}\right)=\frac{F_{X}\left(k_{1-\alpha}\right)-(1-\alpha)}{\operatorname{Pr}\left(X=k_{1-\alpha}\right)}=p^{*}
$$

donde $k_{1-\alpha}$ es el cuantil $1-\alpha$ de la distribución de $X$ bajo la hipótesis nula.

La misma definición de cuantil garantiza que la expresión de la derecha de (6) sea mayor que cero y, considerando que $F_{X}\left(k_{1-\alpha}\right)=F_{X}\left(k_{1-\alpha}-1\right)+\operatorname{Pr}\left(X=k_{1-\alpha}\right)$ y que $F_{X}\left(k_{1-\alpha}-1\right)<1-\alpha$, se muestra que la expresión es menor que 1 . El intervalo $A_{R}$ se puede entonces identificar con $\left[0, p^{*}\right)$.

El procedimiento de aleatorización de la prueba consiste entonces en:

1. Determinar los datos preliminares: el tamaño de la población $N$, el tamaño de la muestra, $n$ y el valor de $N_{0}$ de la hipótesis nula, sin olvidar que estamos considerando la hipótesis alternativa como $H_{1}: N_{A}>N_{0}$.

2. Calcular $p^{*}$ con la expresión dada en (6) .

3. Generar un valor $u$ de una variable aleatoria $U \sim U(0,1)$.

4. La regla de decisión se define en función del valor $u$ obtenido:

- Si $u \leq p^{*}$, se rechaza $H_{0}$ en favor de $H_{1}$ si $X \geq k_{1-\alpha}$. Se incluye el percentil $k_{1-\alpha}$ en la región de rechazo.

- Si $u>p^{*}$, se rechaza $H_{0}$ en favor de $H_{1}$ si $X>k_{1-\alpha}$. No se incluye el percentil $k_{1-\alpha}$ en la región de rechazo.

De $u$ sólo se toma su condición dicotómica $u \leq p^{*}$ o $u>p^{*}$ y, por lo tanto, su distribución puede reemplazarse por una de Bernoulli con parámetro $p^{*}$ y el efecto sobre la aleatorización es el mismo. En este caso, si el valor generado es 1, se incluye el cuantil en la región de rechazo y si es 0 , no.

El procedimiento se realiza antes de obtener los datos de la muestra, pues lo que se aleatoriza es la prueba y no el resultado, aunque la independencia de $U$ con respecto a la muestra permite hacerlo en cualquier momento. Cuando lleguen los datos de la muestra, ya se tendrá definido si el cuantil se encuentra dentro o fuera de la región de rechazo. Los valores de $u$ y de $p^{*}$ son parte de los elementos determinantes de la prueba y se incluyen para que se puedan reconstruir los cálculos. Además, una vez aleatorizada una prueba, se debe respetar la regla de decisión que se aplica a la muestra para la cual fue definida. Sólo se admite una nueva aleatorización para otra prueba diferente.

\subsection{Valor-p aleatorizado}

Los resultados de la prueba pueden presentarse en la forma usual, con el valor-p para tomar la decisión. 
Como la aleatorización sólo afecta la inclusión del cuantil $k_{1-\alpha}$, si el valor observado de la estadistica de prueba $x_{o b s} \neq k_{1-\alpha}$, el valor-p es igual a $\operatorname{Pr}(X \geq$ $x_{o b s}$, pero si $x_{o b s}=k_{1-\alpha}$, se rechaza $H_{0}$ si $u \leq p^{*}$, pero esto es lo mismo que $1-F_{X}\left(k_{1-\alpha}\right)+u \operatorname{Pr}\left(X=k_{1-\alpha}\right) \leq \alpha$. Por lo tanto, el valor-p aleatorizado se define como:

$$
\begin{aligned}
V_{p}= & \left(1-F_{X}\left(x_{o b s}\right)+\operatorname{Pr}\left(X=x_{o b s}\right)\right)\left(1-I_{\left\{k_{1-\alpha}\right\}}\left(x_{o b s}\right)\right)+ \\
& \left(1-F_{X}\left(x_{o b s}\right)+u \operatorname{Pr}\left(X=x_{o b s}\right)\right) I_{\left\{k_{1-\alpha}\right\}}\left(x_{o b s}\right)
\end{aligned}
$$

donde $I_{\{y\}}(x)$ toma el valor 1 si $x \in\{y\}$ y 0 si no, y $x_{o b s}$ es el valor de $X$ que se observa con la muestra. La función Vp_a hace los cálculos para pruebas unilaterales derechas sobre $N_{A}$.

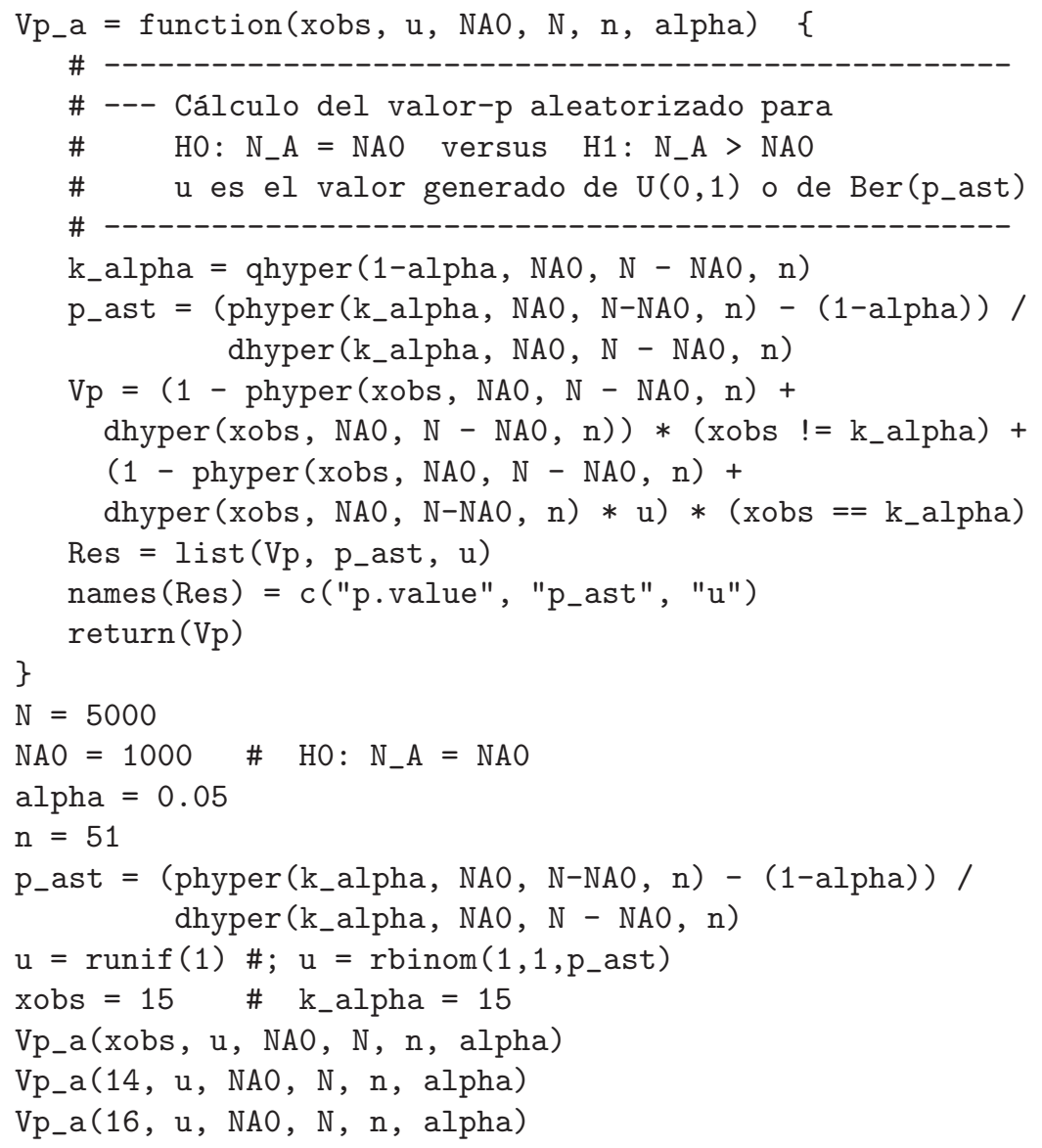

Con el fin de reconstruir los resultados, se fijó la semilla con set.seed(314159) antes de generar el valor de $u$. En este caso se obtuvo 0.2120615 , menor que $p^{*}$ igual a 0.4153287, así que la prueba aleatorizada incluye el cuantil $k_{1-\alpha}=15$ en la región de rechazo. Todavía no se tienen los datos de la muestra aleatoria, 
pero ya sabemos que si se encuentra $x_{o b s}=15$, el valor-p aleatorizado será igual a .04315995 y no el de la tabla, igual a .069675. Esto quiere decir que para esta prueba aleatorizada si se encuentra $x_{o b s}=15$, se rechaza $H_{0}$, mientras que sin la aleatorización, no.

Las simulaciones con el código que sigue muestran el impacto del ajuste del tamaño de la prueba al nivel de significación del investigador. Dentro de las ventajas de la aleatorización se encuentra el incremento consecuente en la función de potencia, calculable además como:

$$
p^{*} \times \operatorname{Pr}\left(X=k_{1-\alpha} \mid H_{1}\right)
$$

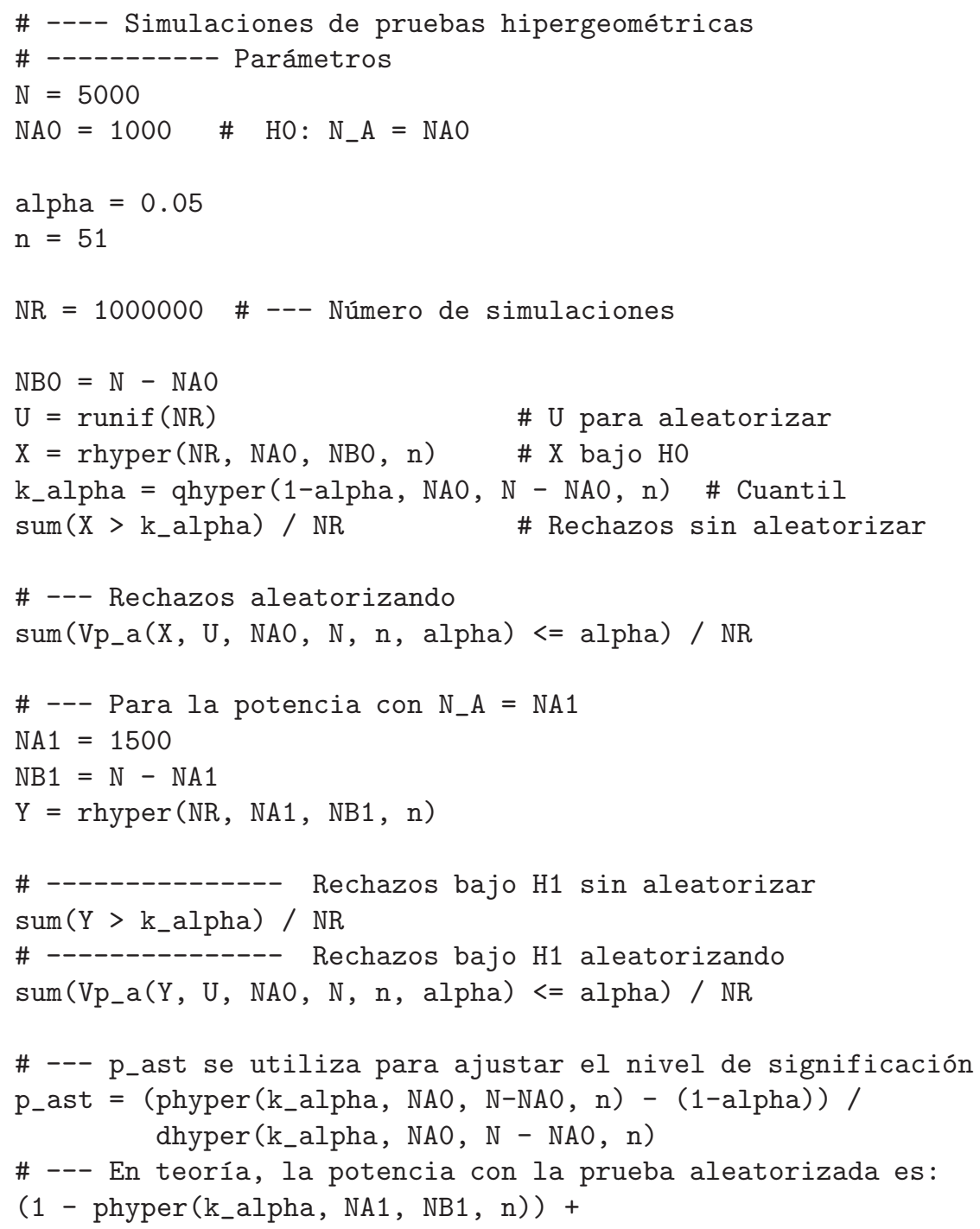


dhyper(k_alpha, NA1, NB1, n) * p_ast

De nuevo, fijando la semilla con set.seed(314159) (sólo para reconstruir los resultados), las simulaciones muestran que la proporción de rechazos de $H_{0}$ es:

- 0.035979 bajo $H_{0}$, sin aleatorizar.

- 0.049857 bajo $H_{0}$, aleatorizando. La diferencia con $\alpha$ se debe a la simulación, pero el efecto de acercar el tamaño de la prueba al nivel de significación de $5 \%$ es claro.

- 0.46784 bajo $H_{1}: N_{A}=1500>1000$, sin aleatorizar.

- 0.51841 bajo $H_{1}: N_{A}=1500>1000$, aleatorizando.

Obsérvese el efecto en la potencia para $N_{A}=1500$. La potencia teórica de 0.467525 , sin aleatorizar, se encuentra en la tabla generada al comienzo de la sección. La diferencia teórica se calcula con (8), lo que da una potencia teórica con el efecto de la aleatorización igual a 0.51817 , muy cercano al obtenido con la simulación.

\section{Conclusiones}

La aleatorización de las pruebas que utilizan estadísticas discretas es un procedimiento sencillo y automatizable que resuelve los problemas de comparabilidad de resultados y de ajuste del tamaño de la prueba al nivel de significación que usualmente se emplea.

Lo que se aleatoriza es la prueba, es decir, la regla de decisión y no el resultado obtenido.

El cambio en la potencia de la prueba es fácilmente calculable a partir de la información utilizada en la aleatorización.

Recibido: 20 de enero de 2011

Aceptado: 30 de marzo de 2011

\section{Referencias}

Bickel, P. J. \& Docksum, K. A. (1977), Mathematical Statistics: Basic Ideas and Selected Topics, John Wiley \& Sons, New York.

Mood, A. F., Graybill, F. A. \& Boes, D. C. (1974), Introduction to the Theory of Statistics, McGraw-Hill, New York.

Comunicaciones en Estadística, junio 2011, Vol. 4, No. 1 
R Development Core Team (2007), R: A Language and Environment for Statistical Computing, R Foundation for Statistical Computing, Vienna, Austria. ISBN 3-900051-07-0.

*http://www.R-project.org

Rohatgi, V. K. (1977), An Introduction to Probability Theory and Mathematical Statistics, John Wiley \& Sons, New York. 